\title{
Modulate Photoinduced Electron Transfer Efficiency of Bipolar Dendritic Systems
}

2008

Vol. 10, No. 15

$3211-3214$

\section{Yu-Hsien Lin, Hsu-Hsuan Wu, Ken-Tsung Wong, ${ }^{*}$ Cheng-Chih Hsieh, Yi-Chih Lin, and Pi-Tai Chou*}

Department of Chemistry, National Taiwan University, Taipei 106, Taiwan

kenwong@ntu.edu.tw; chop@ntu.edu.tw

Received May 12, 2008

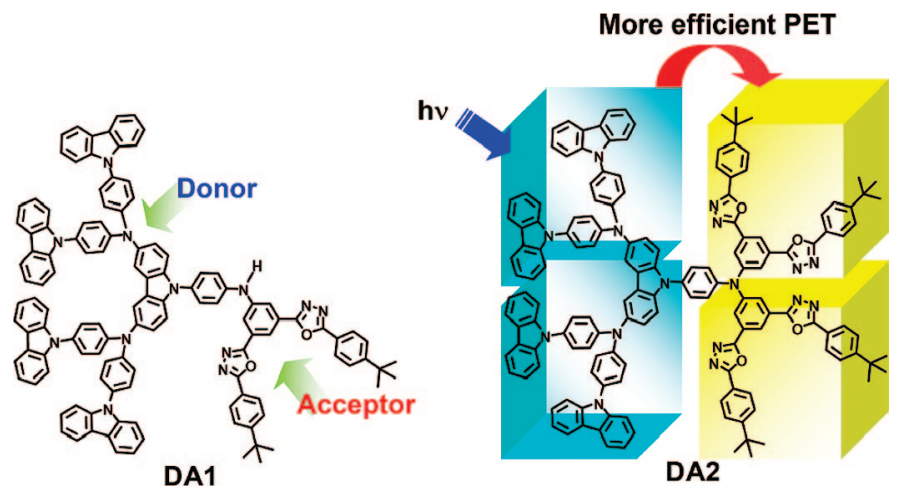

Covalent linkage of dendritic carbazole-based donors and 1,3,4-oxdiazole-based acceptors renders novel bipolar dendrimers that can efficiently facilitate the photoinduced electron transfer (PET) process. Photodynamic studies indicated that the PET rate of bipolar dendrimers DA1 and DA2 can be modulated by the number of acceptors presented in the molecule.

Photoinduced electron transfer (PET) is an essential process for solar energy conversion both in biological photosynthesis and artificial photovoltaic devices. PET occurring in molecular systems can be achieved by a covalently linked donor (D) and acceptor (A) with judicious choice of a bridging system. The rate and efficiency of electron-transfer processes can be controlled over parameters such as distance and spatial orientation between donor and acceptor. In the biological light-harvesting systems, the light-capturing chromophores are assembled into well-organized arrays which then funnel the excitation energy to the photosynthetic center in a very efficient way, triggering the charge separation for subsequent chemical reactions. ${ }^{1}$ Inspired by this natural preorganization process, scientists have made significant endeavors for assembling molecules into an integrated system to mimic the natural photosynthesis center. In this regard, the unique

(1) Pullerits, T.; Sundström, V. Acc. Chem. Res. 1996, 29, 381. molecular architectures of dendrimers provide the essential structural features for the preparation of artificial lightharvesting systems. ${ }^{2}$ Most dendrimers enabled for efficient electron transfer are usually featured with electron-donating dendrons, which serve as antenna located at the peripheral positions of a well-defined electron-accepting core or focal point. The rate and efficiency of PET can thus be manipulated by independent modifications on the chemical structures of donor and acceptor as well as the donor's dendritic generations. ${ }^{3}$ More recently, efficient PET processes can also be achieved in supramolecular arrays formed by noncovalent

(2) (a) Jiang, D.-L.; Aida, T. Nature 1997, 388, 454. (b) Bar-Haim, A.; Klafter, J.; Kopelman, R. J. Am. Chem. Soc. 1997, 119, 6197. (c) Adronov, A.; Frechet, J. M. J. Chem. Commun. 2000, 1701. (d) Choi, M.-S.; Aida, T.; Yamazaki, T.; Yamazaki, I. Angew. Chem., Int. Ed. 2001, 40, 3194. (e) Choi, M.-S.; Aida, T.; Yamazaki, T.; Yamazaki, I. Chem. Eur. J. 2002, 8, 2668. (f) Larsen, J.; Puntoriero, F.; Pascher, T.; McClenaghan, N.; Campagna, S.; Aakesson, E.; Sundstroem, V. ChemPhysChem 2007, 8, 2643. 
interactions between electron-donating dendrimers and electron-accepting chromophores. ${ }^{4}$

Instead of making a funnel-like dendritic system, we have long anticipated that dendrimers incorporated with preorganized donors (donor dendron) for capturing excitation energy and with multiple electron acceptors (acceptor dendron) for trapping the photoexcited electrons could give rise to a new type of bipolar dendrimer with a more efficient PET process. In this paper, we report the synthesis, electrochemical, and phophysical properties of two novel D-A dendrimers (DA1 and DA2, Scheme 1), in which the electron-donating

Scheme 1. Synthesis of Bipolar Dendrimers DA1 and DA2
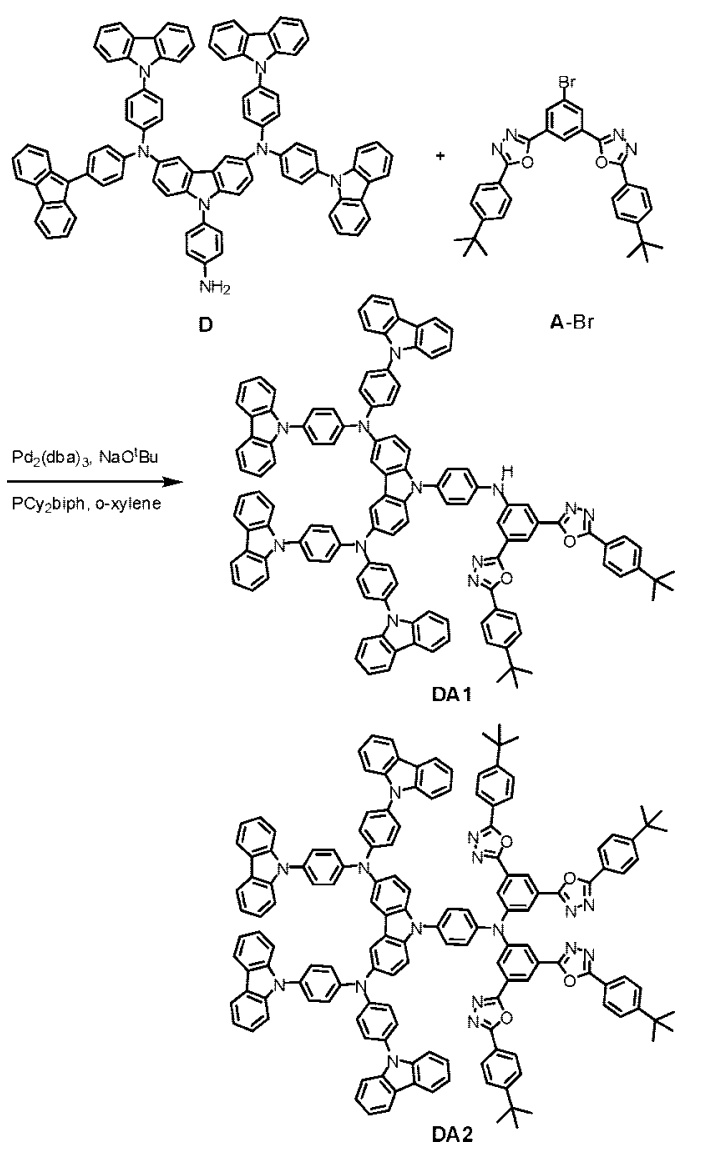

9-phenylcarbazole-based dendron was covalently hybridized with an electron-accepting 1,3,4-oxdiazole-based dendron.

(3) (a) Segura, J. L.; Gómez, R.; Martín, N.; Luo, C.; Swartz, A.; Guldi, D. M. Chem. Commun. 2001, 707. (b) Hahn, U.; Gorka, M.; Vogtle, F.; Vicinelli, V.; Ceroni, P.; Maestri, M.; Balzani, V. Angew. Chem., Int. Ed. 2002, 41, 3595. (c) Cameron, C. S.; Gorman, C. B. Adv. Funct. Mater. 2002, 12, 17. (d) Serin, J. M.; Brousmiche, D. W.; Frechet, J. M. J. Chem. Commun. 2002, 2605. (e) Thomas, K. R. J.; Thompson, A. L.; Sivakumar, A. V.; Bardeen, C. J.; Thayumanavan, S. J. Am. Chem. Soc. 2005, 127 373. (f) Sivanandan, K.; Aathimanikandan, S. V.; Arges, C. G.; Bardeen, C. J.; Thayumanavan, S. J. Am. Chem. Soc. 2005, 127, 2020. (g) Nantalaksakul, A.; Dasari, R. R.; Ahn, T.-S.; Al-Kaysi, R.; Bardeen, C. J.; Thayumanavan, S. Org. Lett. 2006, 8, 2981.

(4) (a) Aathimanikandan, S. V.; Sandanaraj, B. S.; Arges, C. G.; Bardeen, C. J.; Thayumanavan, S. Org. Lett. 2005, 7, 2809. (b) Li, W.-S.; Kim, K. S.; Jiang, D.-L.; Tanaka, H.; Kawai, T.; Kwon, J. H.; Kim, D.; Aida, T. J. Am. Chem. Soc. 2006, 128, 10527.
Carbazole and 1,3,4-oxdiazole were selected as donor and acceptor, respectively, because both of them were ubiquitously exploited in organic electronics for charge transport functions. ${ }^{5}$ Covalent linkage of dendritic donors and acceptors creates a novel bipolar system equipped with multiple donors and acceptors that may facilitate the PET process. Photodynamic studies indicate that the PET rate of bipolar dendrimers DA1 and DA2 can be modulated by the number of acceptors presented in the $\mathrm{D}-\mathrm{A}$ dendrimers.

The synthesis of D-A dendrimers (DA1 and DA2) is depicted in Scheme 1. The 9-phenylcarbazole-based dendron (D) was synthesized by our group previously through efficient $\mathrm{C}-\mathrm{N}$ bond formation reactions. ${ }^{6}$ The acceptor counterpart (A-Br) bearing two 1,3,4-oxadiazole moieties was synthesized according to the reported procedures. ${ }^{7}$ The covalent hybridization of the donor and the acceptor was successfully achieved by a $\mathrm{Pd}$-catalyzed $\mathrm{C}-\mathrm{N}$ bond coupling reaction of 9-phenylcarbazole-based dendron $\mathbf{D}$ with 1,3,4oxdiazole-containing bromideA in the presence of $\operatorname{Pd}_{2}(\mathrm{dba})_{3}$ as a catalyst and 2-dicyclohexylphospine biphenyl as the ligand, yielding DA1 and DA2 in $30 \%$ and 67\%, respectively.

Both of DA1 and DA2 are amorphous materials, exhibiting $T_{\mathrm{g}}$ at 237 and $250^{\circ} \mathrm{C}$, respectively, analyzed by differential scanning calorimetry (DSC), and show high thermal tolerance $\left(T_{\mathrm{d}}>448{ }^{\circ} \mathrm{C}\right)$, analyzed by thermogravimetric analysis (TGA). The bipolar characters of DA1 and DA2 were first probed by cyclic voltammetry (CV). Both compounds exhibit two reversible one-electron oxidation potentials (DA1: 0.25, 0.44; DA2: $0.28,0.46 \mathrm{~V}$ vs ferrocene/ferrocenium ion, $\mathrm{Fc} /$ $\mathrm{Fc}^{+}$) followed by a multiple-electron oxidation peak centered at $0.80 \mathrm{~V}$ (DA2 shown in Figure 1, DA1 shown in Figure S-1, Supporting Information). We assigned the first two peaks to the successive oxidations of the donor's central part: 3,6diaminocarbazole. Upon attaching to the acceptor(s), these two oxidations are slightly shifted to higher potentials as compared to those of the donor-only compound $\mathbf{D}$, which displays the first two oxidation peaks at 0.22 and $0.42 \mathrm{~V}$. The third oxidation potential $(0.80 \mathrm{~V})$ then was ascribed to the oxidations of peripheral carbazoles. DA1 and DA2 showed reversible reduction peaks at -2.47 and $-2.23 \mathrm{~V}$, respectively. The model compound A (see the Supporting Information) also displayed a reversible reduction potential at $-2.42 \mathrm{~V}$. Thus, the cathodic reduction couples can be unambiguously attributed to the reduction process at the phenylene ring containing 1,3,4-oxdiazole moieties. Interestingly, the DA2 has a lower reduction potential compared to

(5) Carbazole: Hu, N.-X.; Xie, S.; Popovic, Z. D.; Ong, B.; Hor, A.-M. Synth. Met. 2000, 111, 421. (b) Thomas, K. R. J.; Lin, J. T.; Tao, Y.-T.; Ko, C.-W. Adv. Mater. 2000, 12, 1949. (c) Zhang, Q.; Hu, Y. F.; Cheng, Y. X.; Su, G. P.; Ma, D. G.; Wang, L. X.; Jing, X. B.; Wang, F. S. Synth. Met. 2003, 137, 1111. (d) Bugatti, V.; Concilio, S.; Iannelli, P.; Piotto, S. P.; Bellone, S.e.; Ferrara, M.; Neitzert, H. C.; Rubino, A.; Della Sala, D.; Vacca, P. Synth. Met. 2006, 156, 13. (e) Oxadiazole: Freeman, A. W.; Koene, S. C.; Malenfant, P. R. L.; Thompson, M. E.; Frechet, J. M. J. J. Am. Chem. Soc. 2000, 122, 12385. (f) Yang, X.; Jaiser, F.; Klinger, S.; Neher, D. Appl. Phys. Lett. 2006, 88, 021107/1. (g) Ichikawa, M.; Kawaguchi, T.; Kobayashi, K.; Miki, T.; Furukawa, K.; Koyama, T.; Taniguchi, Y. J. Mater. Chem. 2006, 16, 221.

(6) Wong, K.-T.; Lin, Y.-H.; Wu, H.-H.; Fungo, F. Org. Lett. 2007, 9, 4531 .

(7) Kraft, A. Liebigs Ann. Recl. 1997, 1463. 


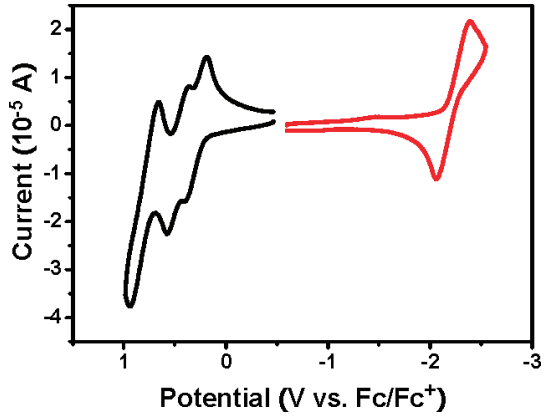

Figure 1. Cyclic voltammogram of compound DA2 (oxidation in $\mathrm{CH}_{2} \mathrm{Cl}_{2}$ and reduction in THF).

that of DA1; this result points out that the LUMO energy of a D-A system can be subtly modulated by the number of acceptors attached. ${ }^{8}$

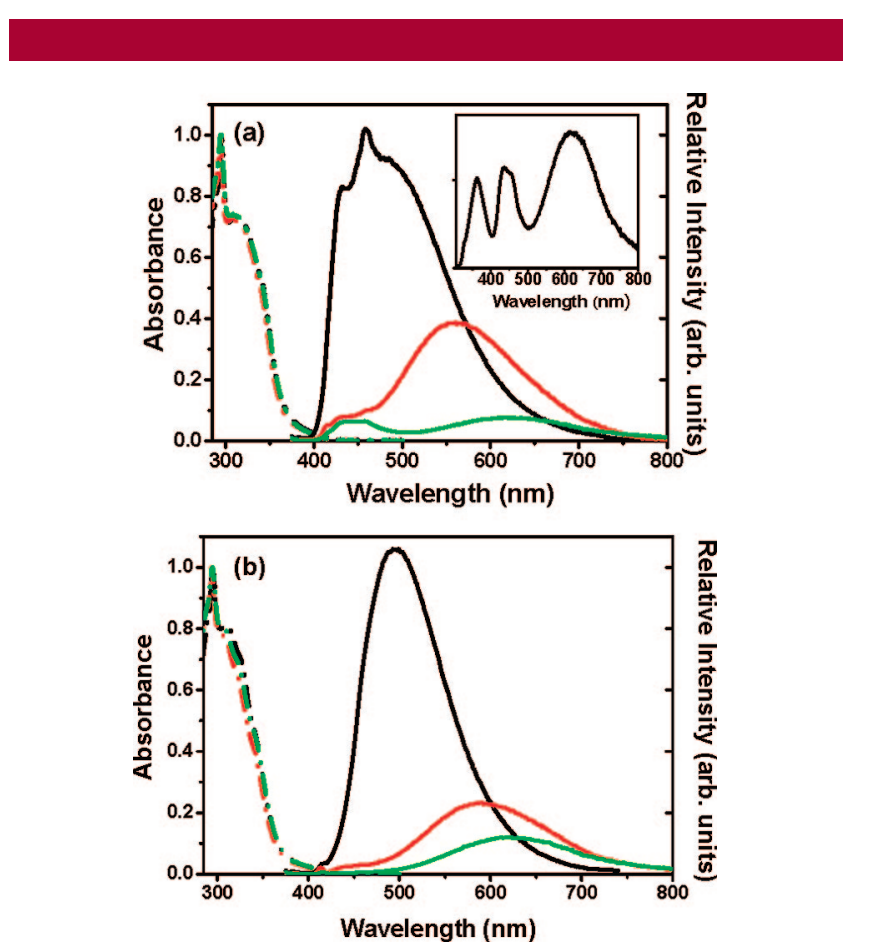

Figure 2. (a) Absorption and emission spectra of DA1 in toluene (black), THF (red), and $\mathrm{CH}_{2} \mathrm{Cl}_{2}$ (green) where $\tau_{\mathrm{ex}}=380 \mathrm{~nm}$. Inset: emission spectrum of DA1 excited at $300 \mathrm{~nm}$ in $\mathrm{CH}_{2} \mathrm{Cl}_{2}$. (b) Absorption and emission spectra of DA2 in toluene (black), THF (red), and $\mathrm{CH}_{2} \mathrm{Cl}_{2}$ (green). All samples are prepared to be $\sim 10^{-5}$ $\mathrm{M}$ in various solvents.

Figure 2 depicts the steady-state absorption and emission spectra of DA1 and DA2 in various solvents. Pertinent spectroscopic and dynamic parameters are listed in Table 1. For both DA1 and DA2, the absorption spectral features could be well convoluted by a linear combination of $\mathbf{D}$ and $\mathbf{A}$ chromophores, indicating a negligibly small interaction (weak

(8) Ku, S.-Y.; Cheng, Y.-M.; Lin, X.-Y.; Hung, Y.-Y.; Pu, S.-C.; Wong, K.-T.; Chou, P.-T.; Lee, G.-H. J. Org. Chem. 2006, 71, 456.
Table 1. Photophysical Properties of D-A Dendrimers DA1 and DA2 in Various Solvents

\begin{tabular}{|c|c|c|c|}
\hline solvent & $\lambda_{\max }(\mathrm{nm})$ & relaxation dynamics ${ }^{a}(\mathrm{~ns})$ & $\Phi$ \\
\hline \multicolumn{4}{|l|}{ DA1 } \\
\hline toluene & 450 & $\begin{array}{c}450 \mathrm{~nm}: \tau_{1}=0.290(0.62) \\
\tau_{2}=10.79(0.38) \\
580 \mathrm{~nm}: \tau_{1}=0.285(-0.33) \\
\tau_{2}=11.36(0.67)\end{array}$ & 0.147 \\
\hline \multirow[t]{2}{*}{ THF } & 435 & $\begin{array}{c}450 \mathrm{~nm}: \tau_{1}=0.068(0.98) \\
\tau_{2}=5.65(0.02)\end{array}$ & 0.047 \\
\hline & 560 & $\begin{array}{c}600 \mathrm{~nm}: \tau_{1}=0.065(-0.67) \\
\tau_{2}=8.95(0.33)\end{array}$ & \\
\hline \multirow[t]{2}{*}{$\mathrm{CH}_{2} \mathrm{Cl}_{2}$} & 435 & $450 \mathrm{~nm}: \tau=0.0594^{b}$ & 0.012 \\
\hline & 620 & $\begin{array}{c}600 \mathrm{~nm}: \tau_{1}=0.0603(-0.36)^{b} \\
\tau_{2}=4.78(0.64)\end{array}$ & \\
\hline \multicolumn{4}{|c|}{ 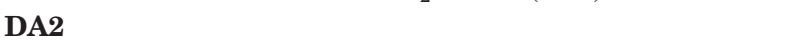 } \\
\hline toluene & 495 & $\begin{array}{c}450 \mathrm{~nm}: \tau_{1}=0.263(0.64) \\
\tau_{2}=12.75(0.36) \\
580 \mathrm{~nm}: \tau_{1}=0.250(-0.22) \\
\tau_{2}=14.18(0.78)\end{array}$ & 0.121 \\
\hline THF & 590 & $\begin{array}{c}450 \mathrm{~nm}: \tau_{1}=0.0021(0.99)^{b} \\
\tau_{2}=15.79(0.01) \\
610 \mathrm{~nm}: \tau=17.70\end{array}$ & 0.026 \\
\hline $\mathrm{CH}_{2} \mathrm{Cl}_{2}$ & 630 & $\begin{array}{c}480 \mathrm{~nm}: \tau_{1}=0.0014(0.80)^{b} \\
\tau_{2}=4.02(0.20) \\
600 \mathrm{~nm}: \tau_{1}=0.0016(-0.28)^{b} \\
\tau_{2}=4.92(0.72)\end{array}$ & 0.012 \\
\hline
\end{tabular}

${ }^{a}$ Data in parentheses is the fitted pre-exponential factor normalized to 1. ${ }^{b}$ The ultrafast relaxation dynamics were measured by a femtosecond up-conversion system.

electronic coupling) between $\mathbf{D}$ and $\mathbf{A}$, so that $\mathbf{D}$ and $\mathbf{A}$ can be treated as separated entities in the ground state. Furthermore, the absorption spectra exhibit nearly solvent polarity independence in the two D-A systems. The similar solvation effect indicates a rather small dipolar change between ground and Franck-Condon excited states. Conversely, the luminescence spectra of these D-A dendrimers show remarkable solventpolarity dependence and reveal multiple emission in polar solvents.

As a prototypical example, DA1 revealed a broad emission band maximized at $\sim 450 \mathrm{~nm}$ in toluene (excitation at $380 \mathrm{~nm}$ ). Upon increasing solvent polarity, the broad emission band (in toluene) gradually separated to dual emission with peak wavelength at $435 \mathrm{~nm}\left(\mathrm{~F}_{1}\right.$ band) and $560 \mathrm{~nm}\left(\mathrm{~F}_{2}\right.$ band $)$ in e.g. THF. While the $F_{1}$ band revealed nearly solvent independence, the peak wavelenght of the $\mathrm{F}_{2}$ band at $560 \mathrm{~nm}$ further redshifted to $620 \mathrm{~nm}$ in $\mathrm{CH}_{2} \mathrm{Cl}_{2}$ (see Table 1). The quantum yield of the entire emission decreased from 0.15 in toluene to 0.01 in $\mathrm{CH}_{2} \mathrm{Cl}_{2}$. The entire emission originating from a common ground-state species is ascertained by the same fluorescence excitation spectra throughout the monitored emission wavelength of 450-700 nm, which are also effectively identical with the absorption spectrum. In yet another approach, the control D and A moieties exhibit solvent independent, normal Stokes shifted emission maxima at 430 and $344 \mathrm{~nm}$, respectively (see Figure S-2, Supporting Information).

Similar solvent-polarity dependent emission was obtained for DA2. In toluene, DA2 revealed a broad emission band with 
peak wavelength at $495 \mathrm{~nm}$ in toluene and dual emission bands upon increasing the solvent polarity to THF and $\mathrm{CH}_{2} \mathrm{Cl}_{2}$, as shown in Figure 2. However, prominent difference can also be highlighted between DA1 and DA2. For comparison, in the same solvents, the intensity ratio for $\mathrm{F}_{1}$ versus $\mathrm{F}_{2}$ band in DA2 was much lower than that of DA1, and $F_{1}$ of DA2 even became obscure in $\mathrm{CH}_{2} \mathrm{Cl}_{2}$. This intriguing observation can be rationalized via probing the corresponding relaxation dynamics for both DA1 and DA2 (vide infra).

As for the origin of the $\mathrm{F}_{2}$ band, we have prepared DA1 and DA2 dendrimers in various solvents with different concentrations of $2 \times 10^{-6}, 1 \times 10^{-5}$, and $1 \times 10^{-4} \mathrm{M}$ to examine the associated photophysical properties. As a result, the absorption spectra, the intensity ratio for $F_{1}$ versus $F_{2}$ bands and the emission profile all showed concentration independence. The results eliminate the possibility that the $\mathrm{F}_{2}$ band originates from the aggregation effect and/or the excimer emission. These, in combination with the cascade type of HOMO/LUMO arrangement between $\mathbf{D}$ and $\mathbf{A}$ (measured by CV, see the Supporting Information), lead us to conclude the occurrence of PET between $\mathbf{D}$ and $\mathbf{A}$, resulting in the $\mathrm{F}_{2}$ emission (see Figure $\mathrm{S}-3$, Supporting Information). Accordingly, the $F_{1}$ band can be unambiguously assigned to the normal emission of the donor moiety. It should be noted that when excitation at the shorter wavelength of $300 \mathrm{~nm}$, in which the transition, in part, is also attributed to the acceptor moiety, DA1 exhibited distinct triple emission, consisting of acceptor $(\sim 360 \mathrm{~nm})$, donor $(450 \mathrm{~nm})$ and the charge transfer emission $(620 \mathrm{~nm})$ in $\mathrm{CH}_{2} \mathrm{Cl}_{2}$. The sum of emission nearly covers the entire range of visible spectrum (see inset of Figure 2a). The resulting multiple emission implies finite rate of PET process, as supported by the following reaction dynamics.

In the steady-state approach, the emission of DA1 in toluene revealed a much broader bandwidth than that of the donor only moiety (Figure S-2, Supporting Information) in the same solvent. We thus suspect that PET is also operative on DA1 (or DA2) in toluene. It is thus crucial to monitor the relaxation dynamics at different wavelength of DA1 in toluene. As shown in Figure 3, the decay traces were drastically different at the very blue side and the red tail of the emission of DA1 (or DA2) in toluene. Details

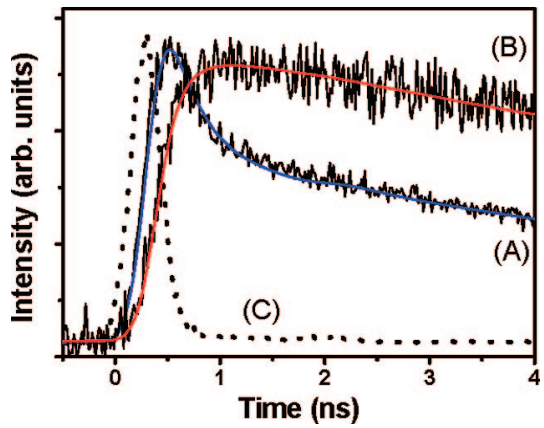

Figure 3. Relaxation dynamics of DA1 in toluene monitored at $298 \mathrm{~K}$ at (A) $450 \mathrm{~nm}$ and (B) $580 \mathrm{~nm}$. (C) The instrument response function of $380 \mathrm{~nm}$. Note that with best curve fitting, the fast decay of $450 \mathrm{~nm}$ ( $290 \mathrm{ps})$ emission correlates very well with the rise of $580 \mathrm{~nm}$ emission (285 ps). of the associated photophysical parameters are listed in Table 1. The decay at $450 \mathrm{~nm}$ can be well fitted by two single exponential components, 290 ps and 10.79 ns. Conversely, the red side at 580 $\mathrm{nm}$ consists of a rise component of $285 \mathrm{ps}$ and a decay of 11.36 ns. The decay of $290 \mathrm{ps}$ at $450 \mathrm{~nm}$, within experimental error, is consistent with the rise ( $285 \mathrm{ps}$ ) monitored at $580 \mathrm{~nm}$, establishing a precursor-successor type of PET process. Furthermore, the identical decay component of $\sim 11 \mathrm{~ns}$ between the two wavelength leads us to conclude the strong overlap of locally excited $\left(\mathrm{F}_{1}\right)$ and electron transfer $\left(\mathrm{F}_{2}\right)$ emission of DA1 in toluene. Upon increasing solvent polarity, a decrease of the $\mathrm{F}_{1}$ intensity implies a faster PET process. This viewpoint was further supported by the relaxation dynamics of $\mathrm{F}_{1}$ band of 68 and 59 ps in THF and $\mathrm{CH}_{2} \mathrm{Cl}_{2}$, respectively (Table 1). Based on the same methodology, we then focused on the relaxation dynamics of DA2 in toluene and also resolved a fast decay (263 ps) and another exceedingly long lifetime (12.75 ns) at short wavelength and a consistent risetime (250 ps) at longer wavelength of DA2 (Table 1). Upon increasing the solvent polariy, PET in DA2 is apparently more facile than that in DA1, as supported by the resolution of faster decay 2.1 and 1.4 ps for DA2 (the F1 band) in THF and $\mathrm{CH}_{2} \mathrm{Cl}_{2}$, respectively. The results qualitatively fit mechanism of PET, in which the charge transfer species is further stabilized with increasing the solvent polarity, resulting in the reduction of solvent-induced barrier and hence the faster reaction rate. ${ }^{9}$ Finally, the relatively long radiative lifetime ( $\gg 10 \mathrm{~ns}$ ) for DA1 and DA2 in various solvents could be rationalized by a meta conjugation between oxdiazole and 4-(9H-carbazol-9-yl)benzenamino moieties. In the excited state, there exists a better communication mechanism through the meta-connectivity than in the ground state. ${ }^{10}$ As a result, a slow charge recombination rate is expected for the charge transfer state (the $\mathrm{F}_{2}$ band).

In conclusion, two bipolar dendritic molecules DA1 and DA2 composed of carbazole-based donors and a different number of 1,3,4-oxadiazole-based acceptors have been synthesized. These two dendritic systems exhibited efficient charge transfer emission, allowing us to probe the detailed electron transfer processes by spectroscopy and the associated relaxation dynamics. The photodynamics indicate that the dendrimer (DA2) equipped with more acceptors can efficiently facilitate the PET process. Our results may spark the demanding for a new molecular design of bipolar dendrimers use for highly efficient light-harvesting systems.

Acknowledgment. This study was supported financially by the National Science Council and Ministry of Education of Taiwan.

Supporting Information Available: Detailed experimental procedures of synthesis and photodynamics measurements; spectroscopic characterization of new compounds; cyclic voltammograms of compounds DA1, D, and A; photophysics of $\mathbf{D}$ and $\mathbf{A}$. This material is available free of charge via the Internet at http://pubs.acs.org.

\section{OL801096C}

(9) Chen, K.-Y.; Hsieh, C.-C.; Cheng, Y.-M.; Lai, C.-H.; Chou, P.-T.; Chow, T.-J. J. Phys. Chem. A 2006, 110, 12136.

(10) (a) Zimmerman, H. E. J. Am. Chem. Soc. 1995, 117, 8988. (b) Thompson, A. L.; Ahn, T.-S.; Thomas, K. R. J.; Thayumanavan, S.; Martinez, T. J.; Bardeen, C. J. J. Am. Chem. Soc. 2005, 127, 16348. 